Pooja Bansal · Mohammad Hasan Shahid

\title{
Bounds of generalized normalized $\delta$-Casorati curvatures for real hypersurfaces in the complex quadric
}

Received: 11 April 2018 / Accepted: 10 September 2018 / Published online: 26 September 2018

(C) The Author(s) 2018

\begin{abstract}
In the present paper, we first characterize real hypersurfaces in the complex quadric $Q^{m}$ by giving an inequality in terms of the scalar curvature and the Mean curvature vector field. We also obtain the condition under which this inequality becomes an equality. Further, we develop two extremal inequalities involving the normalized $\delta$-Casorati curvatures and the extrinsic generalized normalized $\delta$-Casorati curvatures for real hypersurfaces in $Q^{m}$. Finally, we derive the necessary and sufficient condition for the equality in both cases.
\end{abstract}

Mathematics Subject Classification $53 \mathrm{C} 25 \cdot 53 \mathrm{C} 40 \cdot 53 \mathrm{C} 55$

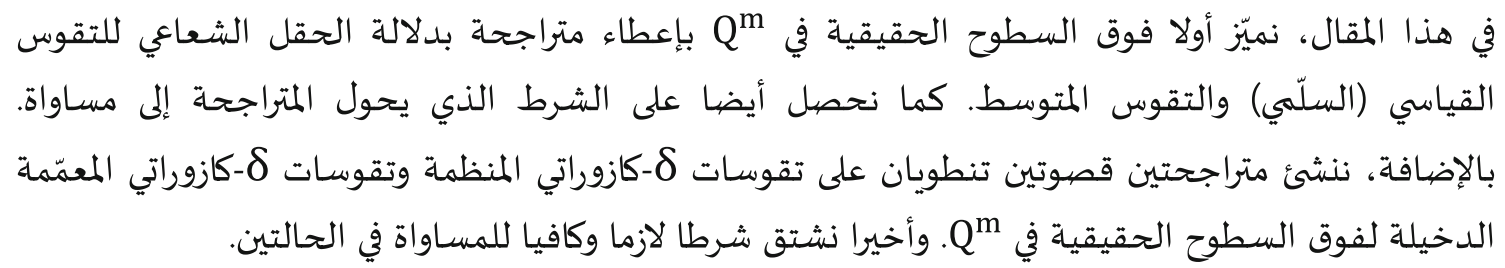

\section{Introduction}

In 1993, Chen [7] introduced the notion of Chen invariants (or $\delta$-invariants) and obtained some optimal inequalities consisting of intrinsic and some extrinsic invariants for any Riemannian submanifold [8].

Moreover, Casorati introduced the theory of the extrinsic invariant given by the normalized square of the second fundamental form, which is known as Casorati curvature of a submanifold in a Riemannian manifold [6]. This theory of Casorati curvature is an extended version of the notion of principal curvatures of a hypersurface in a Riemannian manifold. It is a very fast growing area of research to obtain geometric relations or inequalities concerning the esteemed curvature. Thus, it is both important and very interesting to obtain some extremal inequalities for the Casorati curvatures of submanifolds in any ambient Riemannian manifold.

Additionally, many research articles involving optimal inequalities for the Casorati curvatures have been published by different authors for different submanifolds and ambient spaces in complex as well as in contact

P. Bansal $(\bowtie) \cdot$ M. H. Shahid

Department of Mathematics, Faculty of Natural Sciences, Jamia Millia Islamia, New Delhi 110025, India

E-mail: poojabansal811@gmail.com

M. H. Shahid

E-mail: hasan_jmi@yahoo.com 
geometry. Some optimal inequalities containing Casorati curvatures were obtained for submanifolds of real space forms, complex space forms, Kenmotsu space forms, quaternionic space forms and also for hypersurfaces in complex two-plane Grassmannians and complex hyperbolic two-plane Grassmannians ([9-15, 17,21]). Many geometers studied some geometric and fundamental results on real hypersurfaces of the complex quadric $Q^{m}$ ([1-4,18-20]).

In the present paper, we have characterized the real hypersurfaces in $Q^{m}$ by giving an inequality concerning the scalar curvature and the Mean curvature vector field and proved that the equality holds for a $\mathcal{U}$-principal normal vector field if and only if the real hypersurface $M$ is totally geodesic in $Q^{m}$. Moreover in a continuation we have obtained the optimal inequalities for real hypersurfaces of the complex quadric $Q^{m}$ involving the normalized $\delta$-Casorati curvatures and the extrinsic generalized normalized $\delta$-Casorati curvatures. The equality cases are also considered.

\section{The complex quadric $Q^{m}$}

The complex quadric $Q^{m}$ is the complex hypersurface in $C P^{m+1}$ defined by the relation $z_{1}^{2}+\cdots+z_{m+1}^{2}=0$, where $z_{1}, \ldots, z_{m+1}$ are homogeneous coordinates on $C P^{m+1}$. Then, the Kähler structure on $C P^{m+1}$ naturally induces a standard Kähler structure $(J, g)$ on $Q^{m}$ [18]. The 1-dimensional $Q^{1}$ and 2-dimensional $Q^{2}$ are congruent to the round 2-sphere $S^{2}$ and the Riemannian product $S^{2} \times S^{2}$, respectively. Thus, we will assume that $m$ is greater than or equal to 3 throughout the paper.

Apart from the complex structure $J$, there is one more geometric structure on $Q^{m}$, namely a parallel rank-two vector bundle $\mathcal{U}$ which contains an $S^{1}$-bundle of real structures, that is, complex conjugations $A$ on the tangent spaces of $Q^{m}$. Here the notion of parallel vector bundle $\mathcal{U}$ means that $\left(\bar{\nabla}_{X} A\right) Y=q(X) A Y$ for any vector fields $X$ and $Y$ on $Q^{m}$, where $\bar{\nabla}$ and $q$ denote a connection and a certain 1 -form defined on $T_{p^{\prime}} Q^{m}, p^{\prime} \in Q^{m}$, respectively.

Now, a non-zero tangent vector $W \in T_{p^{\prime}} Q^{m}$ is known as singular tangent vector if it is tangent to more than one maximal flat in $Q^{m}$. There are two types of singular tangent vectors for the complex quadric $Q^{m}$ [20]:

(1) If there exists a complex conjugation $A \in \mathcal{U}$ such that $W \in \mathcal{V}(A)$, then $W$ is singular. Such a singular tangent vector is called $\mathcal{U}$-principal.

(2) If there exists a conjugation $A \in \mathcal{U}$ and orthonormal vectors $U, V \in \mathcal{V}(A)$ such that $W /\|W\|=(U+$ $J V) / \sqrt{2}$, then $W$ is singular. Such a singular tangent vector is called $\mathcal{U}$-isotropic,

where $\mathcal{V}(A)=\left\{X \in T_{\left[p^{\prime}\right]} Q^{m *} \mid A X=X\right\}$ and $J \mathcal{V}(A)=\left\{X \in T_{\left[p^{\prime}\right]} Q^{m *} \mid A X=-X\right\},\left[p^{\prime}\right] \in Q^{m *}$ denote the (+1)-eigenspace and (-1)-eigenspace of the involution $A^{2}=I$ on $T_{\left[p^{\prime}\right]} Q^{m *},\left[p^{\prime}\right] \in Q^{m *}$, respectively.

\section{Some general fundamental equations}

Here, we recall some notions for a real hypersurface $M$ in $Q^{m}$.

Let $M$ be a real hypersurface of $Q^{m}$ with the connection $\nabla$ induced from the Levi-Civita connection $\bar{\nabla}$ in $Q^{m}$. Then

$$
J X=\phi X+\eta(X) N
$$

where $N$ is the unit normal vector field on $M$ and $\phi X$ denotes the tangential component of $J X$ for $X \in \Gamma(T M)$.

Here, $M$ is endowed with an almost contact metric structure $(\phi, \xi, \eta, g)$ satisfying [5]:

$$
\left\{\begin{array}{l}
\xi=-\mathrm{JN}, \eta(\xi)=1, \phi^{2} X=-X+\eta(X) \xi \\
g(\phi X, \phi Y)=g(X, Y)-\eta(X) \eta(Y)
\end{array}\right.
$$

On the other hand, the fundamental Gauss and Weingarten formulas for $M$ are

$$
\begin{aligned}
\bar{\nabla}_{X} Y & =\nabla_{X} Y+h(X, Y)=\nabla_{X} Y+g(S X, Y) N, \\
\bar{\nabla}_{X} N & =-S X,
\end{aligned}
$$


respectively, for $X, Y \in \Gamma(T M)$ and $N \in \Gamma\left(T^{\perp} M\right)$, where $h$ is the second fundamental form and $S$ is the shape operator of $M$, that are related by

$$
g(h(X, Y), N)=g\left(S_{N} X, Y\right)=g(S X, Y) .
$$

Moreover, the structure $(\phi, \xi, \eta, g)$ satisfies

$$
\nabla_{X} \xi=\phi S X
$$

Now, we can choose $A \in \mathcal{U}_{p^{\prime}}$ satisfying $N=\cos (\theta) Z_{1}+\sin (\theta) J Z_{2}$ where $Z_{1}, Z_{2}$ are orthonormal vectors in $\mathcal{V}(A)$ and $0 \leq \theta \leq \frac{\pi}{4}$ (see Proposition 3 [16]) is a function on $M$. Since $\xi=-J N$, we have the following relations:

$$
\begin{aligned}
N=\cos (\theta) Z_{1}+\sin (\theta) J Z_{2}, & A N=\cos (\theta) Z_{1}-\sin (\theta) J Z_{2}, \\
\xi & =\sin (\theta) Z_{2}-\cos (\theta) J Z_{1}, \quad A \xi=\sin (\theta) Z_{2}+\cos (\theta) J Z_{1},
\end{aligned}
$$

from which it follows that $g(\xi, A N)=0$.

Now, from the Gauss equation for $Q^{m} \subset C P^{m+1}$, the Riemannian curvature tensor $R$ of the connection $\nabla$ has the following form [19]:

$$
\begin{aligned}
g(R(X, Y) Z, W)= & g(Y, Z) g(X, W)-g(X, Z) g(Y, W)+g(\phi Y, Z) g(\phi X, W) \\
& -g(\phi X, Z) g(\phi Y, W)-2 g(\phi X, Y) g(\phi Z, W) \\
& +(A Y, Z) g(A X, W)-g(A X, Z) g(A Y, W) \\
& +g(J A Y, Z) g(J A X, W)+g(S Y, Z) g(S X, W) \\
& -g(J A X, Z) g(J A Y, W)-g(S X, Z) g(S Y, W)
\end{aligned}
$$

for all $X, Y, Z, W \in \Gamma(T M)$.

Consider an orthonormal basis $\left\{e_{1}, \ldots, e_{2 m-1}\right\}$ of the tangent space of $M$ and an orthonormal basis $\left\{e_{2 m}=\right.$ $N$ \} of the normal space of $M$. The scalar curvature $\tau$ of $M$ reads

$$
\tau=\sum_{1 \leq i<j \leq 2 m-1} K\left(e_{i} \wedge e_{j}\right)
$$

where $K(\pi)$ stands for the sectional curvature of $M$ associated with a plane section $\pi \subset \Gamma(T M)$ and is spanned by tangent vectors $\left\{e_{i}, e_{j}\right\}$ and $K\left(e_{i} \wedge e_{j}\right)=g\left(R\left(e_{i}, e_{j}\right) e_{j}, e_{i}\right)$ for $1 \leq i<j \leq 2 m-1$.

The normalized scalar curvature $\rho$ of $M$ is given by

$$
\rho=\frac{2 \tau}{(2 m-1)(2 m-2)} .
$$

We denote the mean curvature vector field by $H$, which is given as

$$
H=\frac{1}{2 m-1} \sum_{i=1}^{2 m-1} h\left(e_{i}, e_{i}\right)
$$

Conveniently, we take $h_{i j}^{\alpha}=g\left(h\left(e_{i}, e_{j}\right), e_{\alpha}\right)=g\left(h\left(e_{i}, e_{j}\right), N\right)$ for $i, j \in\{1, \ldots, 2 m-1\}$ and $\alpha=2 m$. Then, one defines the squared mean curvature $\|H\|^{2}$ of $M$ in $Q^{m}$ and the squared norm $\|h\|^{2}$ of the second fundamental form $h$ as:

$$
\|H\|^{2}=\frac{1}{(2 m-1)^{2}}\left(\sum_{i, j=1}^{2 m-1} h_{i j}^{\alpha}\right)^{2} \text { and }\|h\|^{2}=\sum_{i, j=1}^{2 m-1}\left(h_{i j}^{\alpha}\right)^{2},
$$

respectively, where $\alpha=2 m, h_{i j}^{\alpha}=g\left(h\left(e_{i}, e_{j}\right), N\right)$.

It is well known that $\frac{1}{2 m-1}$ times the squared norm of $h$ is called the Casorati curvature $\mathcal{C}$ of $M$ in $Q^{m}$ [6]. Thus, we have

$$
\mathcal{C}=\frac{\|h\|^{2}}{2 m-1}=\frac{1}{2 m-1} \sum_{i, j=1}^{n}\left(h_{i j}^{\alpha}\right)^{2}
$$


Since $\|h\|^{2}=\operatorname{tr}\left(S^{2}\right)$, the above expression can be reexpressed by

$$
\mathcal{C}=\frac{1}{2 m-1} \operatorname{tr}\left(S^{2}\right)
$$

The real hypersurface $M$ of $Q^{m}$ is said to be invariantly quasi-umbilical if there exists a local orthonormal normal frame $\left\{e_{2 m}\right\}$ of $M$ in $Q^{m}$ such that the shape operators $S_{e_{2 m}}$ have an eigenvalue of multiplicity $2 m-2$ for $\alpha=2 m$ and the distinguished eigendirection of $S_{e_{2 m}}$ is the same for $\alpha=2 m$ [21].

Now, let us suppose that $L$ is a $k$-dimensional subspace of $\Gamma(T M), k \geq 2$, such that $\left\{e_{1}, \ldots, e_{k}\right\}$ is an orthonormal basis of $L$. Then, the scalar curvature $\tau(L)$ and the Casorati curvature $\mathcal{C}(L)$ of the $k$-plane $L$ are given by

$$
\tau(L)=\sum_{1 \leq i<j \leq k} K\left(e_{i} \wedge e_{j}\right), \quad \mathcal{C}(L)=\frac{1}{k} \sum_{i, j=1}^{k}\left(h_{i j}^{\alpha}\right)^{2},
$$

respectively.

The normalized $\delta$-Casorati curvatures $\delta_{c}(2 m-2)$ and $\hat{\delta}_{c}(2 m-2)$ of $M$ in $Q^{m}$ are given by [12]

$$
\begin{aligned}
{\left[\delta_{c}(2 m-2)\right]_{p} } & =\frac{1}{2} \mathcal{C}_{p}+\frac{2 m}{2(2 m-1)} \inf (B) \text { and } \\
{\left[\hat{\delta}_{c}(2 m-2)\right]_{p} } & =2 \mathcal{C}_{p}-\frac{2(2 m-1)-1}{2(2 m-1)} \sup (B),
\end{aligned}
$$

where the set $B$ is defined as $B=\{\mathcal{C}(L) \mid L$ : hyperplane of $\Gamma(T M)\}$.

The generalized normalized $\delta$-Casorati curvatures $\delta_{c}(r ; s)$ and $\hat{\delta}_{c}(r ; s)$ of $M$ in $Q^{m}$ for any positive real number $r \neq s(s+1)$ and $A(r, s)=\frac{s(s+1+r)\left[(s+1)^{2}-(s+1)-r\right]}{r(s+1)}$ where $s=2 m-2$ are given as [12]:

$$
\begin{aligned}
& {\left[\delta_{c}(r ; s)\right]_{p}=r \mathcal{C}_{p}+A(r, s) \inf (B), \text { if } 0<r<s(s+1),} \\
& {\left[\hat{\delta}_{c}(r ; s)\right]_{p}=r \mathcal{C}_{p}+A(r, s) \sup (B), \text { if } r>s(s+1) .}
\end{aligned}
$$

From the above two relations, one can note that the generalized normalized $\delta$-Casorati curvatures $\delta_{c}(r ; 2 m-2)$ and $\hat{\delta}_{c}(r ; 2 m-2)$ are the generalized versions of the normalized $\delta$-Casorati curvatures $\delta_{c}(2 m-2)$ and $\hat{\delta}_{c}(2 m-2)$, respectively, by substituting $r$ by $\frac{(2 m-1)(2 m-2)}{2}$ as:

$$
\begin{aligned}
& {\left[\delta_{c}\left(\frac{(2 m-1)(2 m-2)}{2} ; 2 m-2\right)\right]_{p}=(2 m-1)(2 m-2)\left[\delta_{c}(2 m-2)\right]_{p},} \\
& {\left[\hat{\delta}_{c}\left(\frac{(2 m-1)(2 m-2)}{2} ; 2 m-2\right)\right]_{p}=(2 m-1)(2 m-2)\left[\hat{\delta}_{c}(2 m-2)\right]_{p},}
\end{aligned}
$$

for $p \in M$.

\section{Main results}

In this section, we will obtain some extremal inequalities involving the scalar curvature, the normalized scalar curvature and the generalized normalized $\delta$-Casorati curvature for real hypersurfaces $M$ of the complex quadric $Q^{m}$.

To begin with, contracting with respect to $X$ and $W$ in (3.1), the Ricci curvature is given by [19]

$$
\begin{aligned}
\operatorname{Ric}(Y, Z)= & (2 m-1) g(Y, Z)-3 \eta(Y) \eta(Z)-g(A N, N) g(A Y, Z) \\
& -g\left(S^{2} Y, Z\right)+g(A Z, N) g(A N, Y)+g(A \xi, Z) g(A \xi, Y) \\
& +\operatorname{tr}(S) g(S Y, Z)
\end{aligned}
$$




\section{1 $\mathcal{U}$-principal normal vector field}

In this subsection, we will assume $M$ to be a Hopf hypersurface (i.e., for a smooth function $\alpha=g(S \xi, \xi)$ on $M$, one has $S \xi=\alpha \xi$ ) admitting a $\mathcal{U}$-principal unit vector field $N$ satisfying $A N=N$ (or $A \xi=-\xi$ ). Then, after putting $Z=\xi$ in (4.1) and since $S \xi=\alpha \xi$, we obtain

$$
\begin{aligned}
\operatorname{Ric}(Y, \xi) & =(2 m-4) \eta(Y)-2 g(A N, N) g(A Y, \xi)+\operatorname{tr}(S) \alpha \eta(Y)-\alpha^{2} \eta(Y) \\
& =\left(2 m-4+\alpha \operatorname{tr}(S)-\alpha^{2}\right) \eta(Y)-2 g(A N, N) g(A Y, \xi) \\
& =\left(2 m-2+\alpha \operatorname{tr}(S)-\alpha^{2}\right) \eta(Y) .
\end{aligned}
$$

Also from (4.1), we derive

$$
\begin{aligned}
\operatorname{Ric}(\phi Y, \phi Z)= & (2 m-1) g(\phi Y, \phi Z)-g(A N, N) g(A \phi Y, \phi Z) \\
& +g(A \phi Z, N) g(A N, \phi Y)+g(A \xi, \phi Z) g(A \xi, \phi Y) \\
& +\operatorname{tr}(S) g(S \phi Y, \phi Z)-g\left(S^{2} \phi Y, \phi Z\right) .
\end{aligned}
$$

Again, using our assumption and applying the geometric condition of isometric Reeb flow $(\phi S=S \phi)$, we have

$$
\begin{aligned}
\operatorname{Ric}(\phi Y, \phi Z)= & (2 m-1) g(Y, Z)-(2 m-1) \eta(Y) \eta(Z)-g(A \phi Y, \phi Z) \\
& +\operatorname{tr}(S)[g(S Y, Z)-\alpha \eta(Y) \eta(Z)]-\left[g\left(S^{2} Y, Z\right)-\alpha^{2} \eta(Y) \eta(Z)\right] \\
= & (2 m-1) g(Y, Z)-(2 m-1) \eta(Y) \eta(Z)+g(A Y, Z)+\eta(Y) \eta(Z) \\
& +\operatorname{tr}(S)[g(S Y, Z)-\alpha \eta(Y) \eta(Z)]-\left[g\left(S^{2} Y, Z\right)-\alpha^{2} \eta(Y) \eta(Z)\right],
\end{aligned}
$$

where we have used

$$
\begin{aligned}
g(A \phi Y, \phi Z) & =g(A \phi Y, J Z-\eta(Z) N)=g(\phi Y, A J Z) \\
& =-g(J Y, J A Z)-\eta(Y) g(A N, J Z)=-g(Y, A Z)-\eta(Y) \eta(Z) .
\end{aligned}
$$

Finally, we deduce that

$$
\operatorname{Ric}(\phi Y, \phi Z)=\operatorname{Ric}(Y, Z)+2 g(A Y, Z)+\left(\alpha^{2}-\alpha \operatorname{tr}(S)+4-2 m\right) \eta(Y) \eta(Z) .
$$

Moreover, we have

$$
\operatorname{Ric}(Y, \phi Z)-\operatorname{Ric}(\phi Y, Z)=2(2 m-1) g(Y, \phi Z)
$$

\section{$4.2 \mathcal{U}$-isotropic normal vector field}

Here, again we will assume $M$ to be a Hopf hypersurface (i.e., for any smooth function $\alpha=g(S \xi, \xi)$ on $M$, one has $S \xi=\alpha \xi)$ and $M$ admits a $\mathcal{U}$-isotropic unit vector field $N$ satisfying $g(A N, N)=0$. Then, after putting $Z=\xi$ in (4.1) and since $S \xi=\alpha \xi$, we obtain

$$
\operatorname{Ric}(Y, \xi)=\left(2 m-4+\alpha \operatorname{tr}(S)-\alpha^{2}\right) \eta(Y) .
$$

Again, using our assumption together with the condition of isometric Reeb flow $(\phi S=S \phi)$, we have

$$
\begin{aligned}
\operatorname{Ric}(\phi Y, \phi Z)= & (2 m-1) g(Y, Z)-(2 m-1) \eta(Y) \eta(Z)-g(A \xi, Z) g(A \xi, Y) \\
& +g(A N, Z) g(A N, Y)+\operatorname{tr}(S)[g(S Y, Z)-\alpha \eta(Y) \eta(Z)] \\
& -\left[g\left(S^{2} Y, Z\right)-\alpha^{2} \eta(Y) \eta(Z)\right] \\
= & (2 m-1) g(Y, Z)-3 \eta(Y) \eta(Z)+g(A \xi, Z) g(A \xi, Y) \\
& +3 \eta(Y) \eta(Z)+\operatorname{tr}(S)[g(S Y, Z)-\alpha \eta(Y) \eta(Z)] \\
& -\left[g\left(S^{2} Y, Z\right)-\alpha^{2} \eta(Y) \eta(Z)\right]+g(A N, Z) g(A N, Y) \\
& -(2 m-1) \eta(Y) \eta(Z) .
\end{aligned}
$$


Finally, we get

$$
\operatorname{Ric}(\phi Y, \phi Z)=\operatorname{Ric}(Y, Z)+\left(\alpha^{2}-\alpha \operatorname{tr}(S)+4-2 m\right) \eta(Y) \eta(Z) .
$$

Summarizing, we have the following successive results:

Theorem 4.1 The Ricci curvature of a Hopf hypersurface $M$ in $Q^{m}$ satisfies the following:

(a) $\operatorname{Ric}(Y, \xi)=\left(2 m-2+\alpha \operatorname{tr}(S)-\alpha^{2}\right) \eta(Y)$, if the unit normal vector field $N$ is $\mathcal{U}$-principal.

(b) $\operatorname{Ric}(Y, \xi)=\left(2 m-4+\alpha \operatorname{tr}(S)-\alpha^{2}\right) \eta(Y)$, if the unit normal vector field $N$ is $\mathcal{U}$-isotropic.

Theorem 4.2 The Ricci curvature of a Hopf hypersurface $M$ in $Q^{m}$ with isometric Reeb flow satisfies the following:

(a) If the unit normal vector field $N$ is $\mathcal{U}$-principal, then

(1) $\operatorname{Ric}(\phi Y, \phi Z)=\operatorname{Ric}(Y, Z)+2 g(A Y, Z)+\left(\alpha^{2}-\alpha \operatorname{tr}(S)+4-2 m\right) \eta(Y) \eta(Z)$

(2) $\operatorname{Ric}(Y, \phi Z)-\operatorname{Ric}(\phi Y, Z)=2(2 m-1) g(Y, \phi Z)$

(b) If the unit normal vector field $N$ is $\mathcal{U}$-isotropic, then

$$
\operatorname{Ric}(\phi Y, \phi Z)=\operatorname{Ric}(Y, Z)+\left(\alpha^{2}-\alpha \operatorname{tr}(S)+4-2 m\right) \eta(Y) \eta(Z) .
$$

Lemma 4.3 Given a real hypersurface $M$ in $Q^{m}$ with $\phi \mathcal{Q}=\mathcal{Q} \phi$, where $\mathcal{Q}$ is the Ricci operator, one has

$$
\operatorname{Ric}(\phi Y, \phi Z)=\operatorname{Ric}(Y, Z)-\operatorname{Ric}(Y, \xi) \eta(Z) .
$$

Proof We know that the Ricci operator $\mathcal{Q}$ is defined by $\operatorname{Ric}(Y, Z)=g(\mathcal{Q} Y, Z)$. So, we have

$$
\operatorname{Ric}(\phi Y, \phi Z)=g(\mathcal{Q} \phi Y, \phi Z)=-g(\phi \mathcal{Q} \phi Y, Z) .
$$

Now, assume that one has $\phi \mathcal{Q}=\mathcal{Q} \phi$ on $M$, then

$$
\begin{aligned}
\operatorname{Ric}(\phi Y, \phi Z) & =-g\left(\phi^{2} \mathcal{Q} Y, Z\right)=g(\mathcal{Q} Y, Z)-\eta(\mathcal{Q} Y) \eta(Z) \\
& =\operatorname{Ric}(Y, Z)-\operatorname{Ric}(Y, \xi) \eta(Z)
\end{aligned}
$$

which completes the proof.

By setting $Y=Z=e_{i}$ in (4.1), the scalar curvature of a real hypersurface $M$ in the complex quadric $Q^{m}$ has the form

$$
2 \tau=(2 m-1)^{2}+g(A N, N)^{2}-1+(2 m-1)^{2}\|H\|^{2}-\|h\|^{2},
$$

where we have used

$$
\begin{aligned}
\|h\|^{2} & =g\left(h\left(e_{i}, e_{j}\right), h\left(e_{i}, e_{j}\right)\right)=g\left(g\left(S e_{i}, e_{j}\right) N, g\left(S e_{i}, e_{j}\right) N\right) \\
& =\operatorname{tr}\left(S^{2}\right) .
\end{aligned}
$$

Case 1: If the unit normal vector field $N$ is $\mathcal{U}$-principal, then the scalar curvature given by (4.3) has the reduced form

$$
2 \tau=(2 m-1)^{2}\left(1+\|H\|^{2}\right)-\|h\|^{2} .
$$

From this, we have the inequality

$$
\|H\|^{2} \geq \frac{2 \tau}{(2 m-1)^{2}}-1
$$

and equality holds if and only if the real hypersurface $M$ is totally geodesic in $Q^{m}$.

Case 2: If the unit normal vector field $N$ is $\mathcal{U}$-isotropic, then (4.3) reduces to

$$
2 \tau=(2 m-1)^{2}\left(1+\|H\|^{2}\right)-\|h\|^{2}-1
$$

and thus, we have the following inequality

$$
\|H\|^{2} \geq \frac{2 \tau}{(2 m-1)^{2}}-1 .
$$

Consequently, we state 
Theorem 4.4 Let $M$ be a real hypersurface in $Q^{m}$. Then, the scalar curvature and the Mean curvature vector field satisfy

$$
\|H\|^{2} \geq \frac{2 \tau}{(2 m-1)^{2}}-1
$$

and equality holds for a $\mathcal{U}$-principal normal vector field if and only if the real hypersurface $M$ is totally geodesic.

Corollary 4.5 Let $M$ be a totally geodesic real hypersurface of $Q^{m}$. Then, $M$ has constant scalar curvature.

Theorem 4.6 For a real hypersurface $M$ of $Q^{m}$, the generalized normalized $\delta$-Casorati curvatures $\delta_{c}(r ; 2 m-$ 2) and $\hat{\delta}_{c}(r ; 2 m-2)$ satisfy

$$
\begin{aligned}
& \text { (i) } \rho \leq \frac{\delta_{c}(r ; 2 m-2)}{(2 m-1)(2 m-2)}+\frac{g(A N, N)^{2}}{(2 m-1)(2 m-2)}+\frac{2 m}{2 m-1}, \\
& \text { (ii) } \rho \leq \frac{\hat{\delta}_{c}(r ; 2 m-2)}{(2 m-1)(2 m-2)}+\frac{g(A N, N)^{2}}{(2 m-1)(2 m-2)}+\frac{2 m}{2 m-1},
\end{aligned}
$$

respectively. Moreover, both relations (i) and (ii) become equalities if and only if $M$ is an invariantly quasiumbilical real hypersurface having flat normal connection $\nabla^{\perp}$ in $Q^{m}$ such that with respect to some orthonormal basis $\left\{e_{1}, \ldots, e_{2 m-1}\right\}$ and $\left\{e_{2 m}=N\right\}$ of $\Gamma(T M)$ and $\Gamma\left(T^{\perp} M\right)$, respectively, the shape operator $S_{N}$ takes the matrix form

$$
S_{N}=\left(\begin{array}{ll}
\mathcal{M} & 0 \\
0 & \frac{(2 m-1)(2 m-2)}{r} a
\end{array}\right),
$$

where $\mathcal{M}$ is the diagonal matrix of order $2 m-2$ with entries $a$.

Proof In terms of the Casorati curvature, we have the relation

$$
2 \tau=(2 m-1)^{2}+g(A N, N)^{2}-1+(2 m-1)^{2}\|H\|^{2}-(2 m-1) \mathcal{C} .
$$

Consider the quadratic polynomial in the components of the second fundamental form with $n=2 m-1$

$$
\mathcal{P}=r \mathcal{C}+\frac{(n-1)(n+r)\left(n^{2}-n-r\right)}{r n} \mathcal{C}(L)-2 \tau(p)+n^{2}+g(A N, N)^{2}-1 .
$$

Let us assume that $L$ is spanned by $\left\{e_{1}, \ldots, e_{n-1}\right\}$ and take $e_{\alpha}=N=e_{n+1}$ for $\alpha=n+1$. Then, we have

$$
\begin{aligned}
\mathcal{P}= & \frac{r}{n} \sum_{i, j=1}^{n}\left(h_{i j}^{\alpha}\right)^{2}+\frac{(n+r)\left(n^{2}-n-r\right)}{r n} \sum_{i, j=1}^{n-1}\left(h_{i j}^{\alpha}\right)^{2}-2 \tau(p) \\
& +n^{2}+g(A N, N)^{2}-1 .
\end{aligned}
$$

With a simple calculation, we have

$$
\begin{aligned}
\mathcal{P} & =\frac{r}{n} \sum_{i, j=1}^{n}\left(h_{i j}^{\alpha}\right)^{2}+\frac{(n+r)\left(n^{2}-n-r\right)}{r n} \sum_{i, j=1}^{n-1}\left(h_{i j}^{\alpha}\right)^{2}-n^{2}\|H\|^{2}+n \mathcal{C} \\
& =\frac{n+r}{n} \sum_{i, j=1}^{n}\left(h_{i j}^{\alpha}\right)^{2}+\frac{(n+r)\left(n^{2}-n-r\right)}{r n} \sum_{i, j=1}^{n-1}\left(h_{i j}^{\alpha}\right)^{2}-\left(\sum_{i=1}^{n} h_{i i}^{\alpha}\right)^{2}
\end{aligned}
$$


Then, with some computations, we derive

$$
\begin{aligned}
\mathcal{P}= & \sum_{i=1}^{n-1}\left[\left(h_{i i}^{\alpha}\right)^{2}\left(\frac{n^{2}+n(r-1)-2 r}{r}\right)+\left(\frac{n+r}{n}\right)\left(\left(h_{i n}^{\alpha}\right)^{2}+\left(h_{n i}^{\alpha}\right)^{2}\right)\right] \\
& +\frac{(n-1)(n+r)}{n} \sum_{1 \leq i \neq j \leq n-1}\left(h_{i j}^{\alpha}\right)^{2}-2 \sum_{1 \leq i \neq j \leq n} h_{i i}^{\alpha} h_{j j}^{\alpha}+\frac{r}{n}\left(h_{n n}^{\alpha}\right)^{2} .
\end{aligned}
$$

Now, the critical points $h^{c}=\left(h_{11}^{\alpha}, h_{22}^{\alpha}, \ldots, h_{n n}^{\alpha}\right)$ of $\mathcal{P}$ are the solutions of the system of linear homogeneous equations:

$$
\left\{\begin{array}{l}
\frac{\partial \mathcal{P}}{\partial h_{i i}^{\alpha}}=\frac{2(n+r)(n-1)}{r} h_{i i}^{\alpha}-2 \sum_{k=1}^{n} h_{k k}^{\alpha}=0, \\
\frac{\partial \mathcal{P}}{\partial h_{i n}^{\alpha}}=\frac{2(n+r)}{n} h_{i n}^{\alpha}=0, \\
\frac{\partial \mathcal{P}}{\partial h_{n i}^{\alpha}}=\frac{2(n+r)}{n} h_{n i}^{\alpha}=0, \\
\frac{\partial \mathcal{P}}{\partial h_{i j}^{\alpha}}=\frac{2(n+r)(n-1)}{n} h_{i j}^{\alpha}=0, \\
\frac{\partial \mathcal{P}}{\partial h_{n n}^{\alpha}}=\frac{2 r}{n} h_{n n}^{\alpha}-2 \sum_{k=1}^{n-1} h_{k k}^{\alpha}=0,
\end{array}\right.
$$

for $i, j \in\{1,2, \ldots, n-1\}$ with $i \neq j$.

So, from (4.8) it follows that any solution satisfies $h_{i j}^{\alpha}=0$ for $i \neq j \in\{1,2, \ldots, n\}$.

Moreover, the Hessian matrix of the system (4.8) has the following form:

$$
\mathcal{H}(p)=\left(\begin{array}{lll}
\mathcal{H}_{1} & \mathbf{0} & \mathbf{0} \\
\mathbf{0} & \mathcal{H}_{2} & \mathbf{0} \\
\mathbf{0} & \mathbf{0} & \mathcal{H}_{3}
\end{array}\right),
$$

whose diagonal block submatrices are given by

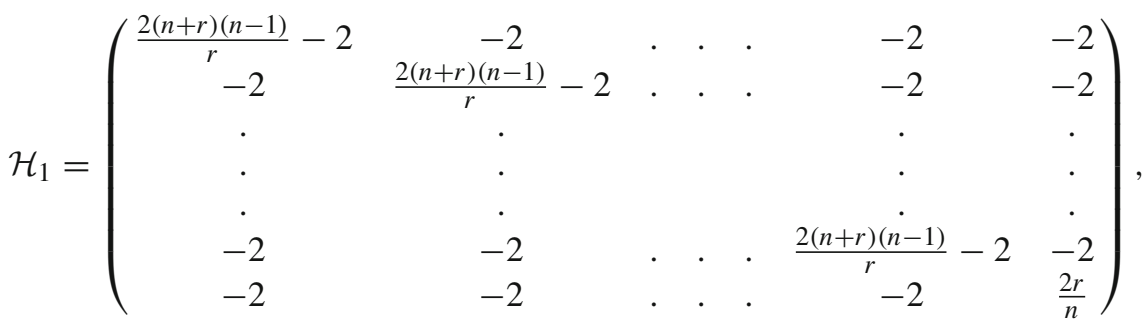

$$
\begin{aligned}
& \mathcal{H}_{2}=\left(\begin{array}{cc}
\frac{2(n+r)(n-1)}{r} & \mathbf{0} \\
\mathbf{0} & \frac{2(n+r)(n-1)}{r}
\end{array}\right), \quad \mathcal{H}_{3}=\left(\begin{array}{cc}
\frac{2(n+r)}{n} & \mathbf{0} \\
\mathbf{0} & \frac{2(n+r)}{n}
\end{array}\right) .
\end{aligned}
$$

Since the Hessian $\mathcal{H}(p)$ is positive semidefinite for all points, the function or the polynomial $\mathcal{P}$ is convex. Due to the convexity of $\mathcal{P}$, the critical point $h^{c}$ is a minimum and in fact a global minimum. Thus, the polynomial $\mathcal{P}$ is of parabolic type and has a minimum at any solution $h^{c}$ of (4.8).

After this, applying (4.8) on (4.7) follows that $h^{c}$ is a solution of $P$, i.e. $\mathcal{P}\left(h^{c}\right)=0$.

So, $\mathcal{P} \geq 0$ and thus from (4.6), we obtain

$$
2 \tau(p) \leq r \mathcal{C}+\frac{(n-1)(n+r)\left(n^{2}-n-r\right)}{r n} \mathcal{C}(L)+n^{2}+g(A N, N)^{2}-1 .
$$

Finally, we have

$$
\rho \leq \frac{r}{n(n-1)} \mathcal{C}+\frac{(n+r)\left(n^{2}-n-r\right)}{r n^{2}} \mathcal{C}(L)+\frac{n+1}{n}+\frac{g(A N, N)^{2}}{n(n-1)} .
$$

Similarly, one can easily get the inequality (ii). 
Furthermore, we can easily check that the equality arises in the theorem if and only if

$$
\begin{aligned}
& h_{i j}=0 \text { for } i, j \in\{1,2, \ldots, n\} \text { with } i \neq j, \\
& h_{n n}=\frac{n(n-1)}{r} h_{11}=\frac{n(n-1)}{r} h_{22}=\cdots=\frac{n(n-1)}{r} h_{n-1} n-1 .
\end{aligned}
$$

Thus, we get the equality case if and only if the real hypersurface $M$ is invariantly quasi-umbilical having flat normal connection $\nabla^{\perp}$ in $Q^{m}$ such that the shape operator takes the form (4.4).

Corollary 4.7 Let $M$ be a real hypersurface of $Q^{m}$. Then

(i) the generalized normalized $\delta$-Casorati curvature $\delta_{c}(r ; 2 m-2)$ for a $\mathcal{U}$-principal (resp. $\mathcal{U}$-isotropic) normal vector field satisfies

$$
\rho \leq \frac{\delta_{c}(r ; 2 m-2)}{(2 m-1)(2 m-2)}+\frac{2 m-1}{2 m-2}\left(\text { resp. } \rho \leq \frac{\delta_{c}(r ; 2 m-2)}{(2 m-1)(2 m-2)}+\frac{2 m}{2 m-1}\right) .
$$

(ii) the generalized normalized $\delta$-Casorati curvature $\hat{\delta}_{c}(r ; 2 m-2)$ for a $\mathcal{U}$-principal (resp. $\mathcal{U}$-isotropic) normal vector field satisfies

$$
\rho \leq \frac{\hat{\delta}_{c}(r ; 2 m-2)}{(2 m-1)(2 m-2)}+\frac{2 m-1}{2 m-2}\left(\text { resp. } \rho \leq \frac{\hat{\delta}_{c}(r ; 2 m-2)}{(2 m-1)(2 m-2)}+\frac{2 m}{2 m-1}\right) .
$$

Moreover, both relations ( $i$ ) and (ii) become equalities if and only if $M$ is an invariantly quasi-umbilical real hypersurface having flat normal connection $\nabla^{\perp}$ in $Q^{m}$ such that with respect to some orthonormal basis $\left\{e_{1}, \ldots, e_{2 m-1}\right\}$ and $\left\{e_{2 m}=N\right\}$ of $\Gamma(T M)$ and $\Gamma\left(T^{\perp} M\right)$ respectively, the shape operator $S_{N}$ takes the following form:

$$
S_{N}=\left(\begin{array}{cc}
\mathcal{M} & 0 \\
0 & \frac{(2 m-1)(2 m-2)}{r} a
\end{array}\right),
$$

where $\mathcal{M}$ is the diagonal matrix of order $2 m-2$ with entries $a$.

Lemma 4.8 For a real hypersurface $M$ of $Q^{m}$, we have

(i) the normalized $\delta$-Casorati curvature $\delta_{c}(2 m-2)$ satisfies

$$
\rho \leq \delta_{c}(2 m-2)+\frac{2 m}{2 m-1}+\frac{g(A N, N)^{2}}{(2 m-1)(2 m-2)} .
$$

Moreover, relation ( $i$ ) becomes an equality if and only if $M$ is an invariantly quasi-umbilical real hypersurface having flat normal connection $\nabla^{\perp}$ in $Q^{m}$ such that with respect to some orthonormal basis $\left\{e_{1}, \ldots, e_{2 m-1}\right\}$ and $\left\{e_{2 m}=N\right\}$ of $\Gamma(T M)$ and $\Gamma\left(T^{\perp} M\right)$ respectively, the shape operator $S_{N}$ takes the following form:

$$
S_{N}=\left(\begin{array}{cc}
\mathcal{M} & 0 \\
0 & a
\end{array}\right)
$$

where $\mathcal{M}$ is the diagonal matrix of order $2 m-2$ with entries $2 a$.

(ii) the normalized $\delta$-Casorati curvature $\hat{\delta}_{c}(2 m-2)$ satisfies

$$
\rho \leq \hat{\delta}_{c}(2 m-2)+\frac{2 m}{2 m-1}+\frac{g(A N, N)^{2}}{(2 m-1)(2 m-2)} .
$$

Moreover, relation (ii) becomes an equality if and only if $M$ is an invariantly quasi-umbilical real hypersurface having flat normal connection $\nabla^{\perp}$ in $Q^{m}$ such that with respect to some orthonormal basis $\left\{e_{1}, \ldots, e_{2 m-1}\right\}$ and $\left\{e_{2 m}=N\right\}$ of $\Gamma(T M)$ and $\Gamma\left(T^{\perp} M\right)$, respectively, the shape operator $S_{N}$ takes the form:

$$
S_{N}=\left(\begin{array}{cc}
\mathcal{M} & 0 \\
0 & 2 a
\end{array}\right)
$$

where $\mathcal{M}$ is the diagonal matrix of order $2 m-2$ with entries $a$. 
Proof From (3.4) (resp. (3.5)) by taking $r=\frac{(2 m-1)(2 m-2)}{2}$ and using (3.2) (resp. (3.3)), we obtain our result for normalized $\delta$-Casorati curvature of a real hypersurface $M$ in $Q^{m}$.

Corollary 4.9 Let $M$ be a real hypersurface of $Q^{m}$.

(i) The normalized $\delta$-Casorati curvature $\delta_{c}(2 m-2)$ for a $\mathcal{U}$-principal normal (resp. $\mathcal{U}$-isotropic) vector field satisfies

$$
\rho \leq \delta_{c}(2 m-2)+\frac{2 m-1}{2 m-2}\left(\text { resp. } \rho \leq \delta_{c}(2 m-2)+\frac{2 m}{2 m-1}\right) .
$$

Moreover, relation ( $i$ ) becomes an equality if and only if $M$ is an invariantly quasi-umbilical real hypersurface having flat normal connection $\nabla^{\perp}$ in $Q^{m}$ such that with respect to some orthonormal basis $\left\{e_{1}, \ldots, e_{2 m-1}\right\}$ and $\left\{e_{2 m}=N\right\}$ of $\Gamma(T M)$ and $\Gamma\left(T^{\perp} M\right)$ respectively, the shape operator $S_{N}$ takes the following form

$$
S_{N}=\left(\begin{array}{cc}
\mathcal{M} & 0 \\
0 & a
\end{array}\right)
$$

where $\mathcal{M}$ is the diagonal matrix of order $2 m-2$ with entries $2 a$.

(ii) The normalized $\delta$-Casorati curvature $\hat{\delta}_{c}(2 m-2)$ for a $\mathcal{U}$-principal (resp. $\mathcal{U}$-isotropic) normal vector field satisfies

$$
\rho \leq \hat{\delta}_{c}(2 m-2)+\frac{2 m-1}{2 m-2}\left(\text { resp. } \rho \leq \hat{\delta}_{c}(2 m-2)+\frac{2 m}{2 m-1}\right) .
$$

Moreover, relation (ii) becomes an equality if and only if $M$ is an invariantly quasi-umbilical real hypersurface having flat normal connection $\nabla^{\perp}$ in $Q^{m}$ such that with respect to some orthonormal basis $\left\{e_{1}, \ldots, e_{2 m-1}\right\}$ and $\left\{e_{2 m}=N\right\}$ of $\Gamma(T M)$ and $\Gamma\left(T^{\perp} M\right)$, respectively, the shape operator $S_{N}$ takes the following form:

$$
S_{N}=\left(\begin{array}{cc}
\mathcal{M} & 0 \\
0 & 2 a
\end{array}\right),
$$

where $\mathcal{M}$ is the diagonal matrix of order $2 m-2$ with entries a.

\section{Further studies}

Here, we present some open problems. Similar problems can be formulated in some different situations, where the expressions of the curvature tensor have some well-known different form.

\subsection{Problem}

To study different submanifolds of the complex quadric $Q^{m}$ and obtain Casorati's inequalities for the same ambient manifold.

\subsection{Problem}

To study slant submanifolds of the complex quadric $Q^{m}$ and obtain Casorati's inequalities for the same ambient manifold, if possible.

\subsection{Problem}

To obtain Casorati's inequalities for submanifolds of locally conformal Kähler space forms. 


\subsection{Problem}

To study some different connections like semi-symmetric non-metric connections and obtain Casorati's inequalities for submanifolds of the complex quadric $Q^{m}$ equipped with semi-symmetric non-metric connection.

Open Access This article is distributed under the terms of the Creative Commons Attribution 4.0 International License (http:// creativecommons.org/licenses/by/4.0/), which permits unrestricted use, distribution, and reproduction in any medium, provided you give appropriate credit to the original author(s) and the source, provide a link to the Creative Commons license, and indicate if changes were made.

\section{References}

1. Bansal, P.: Hopf Real Hypersurfaces in the Complex Quadric $Q^{m}$ with Recurrent Jacobi Operator. Springer, Accepted in AISC series (2017)

2. Bansal, P.; Shahid, M.H.: Optimization Approach for Bounds Involving Generalised Normalised $\delta$-Casorati Curvatures. In: Advances in Intelligent Systems and Computing, vol. 741, pp. 227-237, Springer (2018)

3. Bansal, P., Uddin, S., Shahid, M.H.: Extremities involving B. Y. Chen's invariants for real hypersurfaces in complex quadrics. Accepted in Int. Electron. J. Geom. (2018)

4. Berndt, J.; Suh, Y.J.: Real hypersurfaces with isometric Reeb flow in complex quadrics. Int. J. Math. 24(7), 1350050 (2013)

5. Blair, D.E.: Contact manifolds in Riemannian Geometry. Lecture Notes in Math, vol. 509. Springer, Berlin (1976)

6. Casorati, F.: Mesure de la courbure des surfaces suivant l'idée commune, Ses rapports avec les mesures de courbure gaussienne et moyenne. Acta Math. 14(1), 95-110 (1890)

7. Chen, B.Y.: Some pinching and classification theorems for minimal submanifolds. Arch. Math. (Bassel) 60(6), 568-578 (1993)

8. Chen, B.Y.: An optimal inequality for CR-warped products in complex space forms involving CR $\delta$-invariants. Int. J. Math. 23(3), 1250045 (2012). (17 pages)

9. Chen, B.Y.; Dillen, F.; Van der Veken, J.; Vrancken, L.: Curvature inequalities for Lagrangian submanifolds: the final solution. Differ. Geom. Appl. 31(6), 808-819 (2013)

10. Decu, S.; Haesen, S.; Verstraelen, L.: Optimal inequalities involving Casorati curvatures. Bull. Transilv. Univ. Brasov. Ser. B 14, 85-93 (2007). (Supp. 1)

11. Ghisoiu, V.: Inequalities for the Casorati curvatures of slant submanifolds in complex space forms, Riemannian geometry and applications. In: Proceedings RIGA, ed. Univ. Bucuresti, Bucharest, vol 2011, pp. 145-150 (2011)

12. Lee, J.W.; Vilcu, G.E.: Inequalities for generalized normalized $\delta$-Casorati curvatures of slant submanifolds in quaternionic space forms. Taiwan. J. Math. 19(3), 691-702 (2015)

13. Lee, C.W.; Lee, J.W.; Vilcu, G.E.: A new proof for some optimal inequalities involving generalized normalized $\delta$-Casorati curvatures. J. Inequal. Appl. 2015, 310 (2015). https://doi.org/10.1186/s13660-015-0831-0

14. Lee, C.W.; Lee, J.W.; Vilcu, G.E.: Optimal inequalities for the normalised $\delta$-Casorati curvatures of submanifolds in Kenmotsu space forms. Adv. Geom. 17(3), 355-362 (2017)

15. Park, K.S.: Inequalities for the Casorati curvatures of real hypersurfaces in some Grassmannians. Taiwan. J. Math. 22(1), 63-77 (2018)

16. Reckziegel, H.: On the geometry of the complex quadric. In: Geometry and Topology of Submanifolds VIII (Brussels/Nordfjordeid 1995), pp. 302-315. World Science Publishing, River Edge, NJ (1995)

17. Slesar, V.; Sahin, B.; Vilcu, G.E.: Inequalities for the Casorati curvatures of slant submanifolds in quaternionic space forms. J. Inequal. Appl. 2014, 123 (2014)

18. Suh, Y.J.: Real hypersurfaces in the complex quadric with Reeb parallel shape operator. Int. J. Math. 25(6), 1450059 (2014). (pp. 17)

19. Suh, Y.J.: Real hypersurfaces in the complex quadric with parallel Ricci tensor. Adv. Math. 281, 886-905 (2015)

20. Suh, Y.J.: Psuedo-Einstein real hypersurfaces in the complex quadric. Math. Nachr. 290(11-12), 1884-1904 (2017)

21. Zhang, P.; Zhang, L.: Inequalities for Casorati curvatures of submanifolds in real space forms. Adv. Geom. 16(3), 329-335 (2016)

Publisher's Note Springer Nature remains neutral with regard to jurisdictional claims in published maps and institutional affiliations. 